\title{
NON LINEAR EQUATION OF STATE AND EFFECTIVE PHANTOM DIVIDE IN BRANE MODELS
}

\author{
Jibitesh Dutta ${ }^{1,3 *}$, Subenoy Chakraborty ${ }^{2 \dagger}$ and M. Ansari ${ }^{3 \ddagger}$ \\ ${ }^{1}$ Department of Basic Sciences and Social Sciences, \\ North Eastern Hill University, NEHU Campus, Shillong - 793022 ( INDIA ) \\ ${ }^{2}$ Department of Mathematics, Jadavpur University, Kolkata-32, ( INDIA) and \\ ${ }^{3}$ Department of Mathematics, North Eastern Hill University, \\ NEHU Campus, Shillong - 793022 ( INDIA )
}

(Dated: October 29, 2018)

\begin{abstract}
Here, DGP model of brane-gravity is analyzed and compared with the standard general relativity and Randall-Sundrum cases using non-linear equation of state. Phantom fluid is known to violate the weak energy condition. In this paper, it is found that this characteristic of phantom energy is affected drastically by the negative brane-tension $\lambda$ of the RS-II model. It is found that in DGP model strong energy condition(SEC) is always violated and the universe accelerates only where as in RS-II model even SEC is not violated for $1<\rho / \lambda<2$ and the universe decelerates.
\end{abstract}

PACS numbers: $98.80 . \mathrm{Cq}$

Keywords : DGP brane-world; RS-II model; non-linear equation of state; phantom cosmology; acceleration and deceleration.

\section{INTRODUCTION}

Astrophysical observations made during last few years have shown conclusive evidence for cosmic acceleration at small red-shift 1 3]. It shows beginning of accelerate expansion in the recent past. It is found that cosmic acceleration is driven by some invisible fluid having its gravitational effect in the very

\footnotetext{
* jdutta29@gmail.com, jibitesh@nehu.ac.in

† schakraborty@math.jdvu.ac.in

‡ mansarinehu@gmail.com
} 
late universe. This unknown fluid has distinguishing feature of violating strong energy condition(SEC) being called dark energy [5]. Of late, it was argued that observations are better fit for equation of state parameter $\omega=p / \rho$ ( $p$ being the pressure and $\rho$ being the energy density) lying in a narrow strip around $\omega=-1$ and more towards $\omega<-1$ leading to violation of the weak energy condition (WEC) too [7, $[$ ]]. This dark energy fluid is popularly known as phantom. Using general relativity(GR) based Friedmann equation, giving cosmic dynamics it is found that phantom dominated era of the universe accelerates but ends up in big-rip(BR) singularity after a finite time in future [5]. Later on in [6] using branegravity(BG), singularity free phantom driven cosmic acceleration was obtained. Also, observations support homogeneous and flat model of the universe [4]. The phantom model explains the present and future acceleration of the universe, but it is plagued with the problem of big-rip singularity ( singularity in finite future time when energy density, pressure and the scale factor diverge). Thus, phantom was another exotic matter suggested by Caldwell. Different sources of exotic matter violating SEC [9 18] and WEC were proposed in the recent past [7, 8, 19 33]. A comprehensive review of these contributions is available in [5].

In the race to investigate a viable cosmological model, satisfying observational constraints and explaining present cosmic acceleration, brane-gravity was also drawn into service and brane-cosmology was developed. A review on brane-gravity and its various applications with special attention to cosmology is available in 34 37]. In brane world scenario, our four dimensional universe (a brane ) is a hypersurface embedded in higher dimensional bulk spacetime. In this brane-bulk scenario, all matter and gauge interactions (described by open strings) are localised on a brane while gravity (described by closed strings) may propagate into whole space time. This means that gravity is fundamentally a higher dimensional interaction and we only see the effective 4D theory on brane.

Among the different brane proposals for brane models, the two prominent are RS (proposed by Randall and Sundrum) and DGP(proposed by Dvali, Gabadaze and Porrati) models. In RS models, the hierarchy problem could be solved by a warped or curved extra dimension showing that fundamental scale could be brought down from the Planck scale to $100 \mathrm{GeV}$. Thus, Randall-Sundrum approach brought the theory to scales below $100 \mathrm{GeV}$ being the electroweak scale( so far results could be verified experimentally upto this scale only). In this model, extra-dimension is large having $(1+3)$-branes at its ends. These branes are $Z_{2}$-symmetric (have mirror symmetry) and have tension to counter the negative cosmological constant in the "bulk", which is $\mathrm{AdS}_{5}$. The model, having two (1+3)-branes at the ends of the orbifold $S^{1} / Z_{2}$, is known as RS-I model [38] whereas the model having only one $(1+3)$ - 
brane is known as RS-II model 39|. In particular, RS-II model got much attention due to its simple and rich conceptual base [43 50, 52]. In case, the extra-dimension is time-dependent, brane-gravity induced Friedmann equation (giving dynamics of the universe) contains a correction term $-4 \pi G \rho^{2} / 3 \lambda$ with $\lambda$ being the brane-tension [34 37] . In RS-I model, $\lambda$ is positive, whereas $\lambda$ is negative in RS-II model.

The main idea of the DGP model is the inclusion of a fourdimensional Ricci- Scalar into the action. On the 4-dimensional brane the action of gravity is proportional to $M_{P}^{2}$ whereas in the bulk it is proportional to the corresponding quantity in 5-dimensions. The model is then characterized by a cross over length scale

$$
r_{c}=\frac{M_{P}^{2}}{2 M_{5}^{2}}
$$

such that gravity is 4-dimensional theory at scales $a \ll r_{c}$ where matter behaves as pressure less dust but gravity leaks out into the bulk at scales $a \gg r_{c}$ and matter approaches the behaviour of a cosmological constant [40-42].

In the standard cosmology RS gravity modifies the early universe where as DGP gravity modifies the late universe. So in the present universe brane corrections are not effective in RS-II model. But as phantom energy increases with the expansion, it brings drastic changes in the RS-II model with the equation of state (EOS) given by $p=-\rho-f(\rho)$ with $f(\rho)$ being non-linear functional of $\rho$.

In our recent paper taking the above EOS we have analysed in RS-II model and effective phantom divide is obtained in three cases. So it is natural to study the effect of this EOS in DGP model. The aim of present paper is to extend the work of [6] in DGP model and compare with corresponding solution in RS-II model and GR cases. Further this EOS has been extensively analysed by various authors in GR framework [53 55]. So it is imperative to study this EOS in Brane world scenario. This paper is organised as follows. In sec.2 we give the basic equations of GR, RS-II model and DGP model while in section 3, effective EOS is obtained with brane-gravity corrections and contains discussion on acceleration and deceleration of the models. Section 4 summarizes the work.

\section{BASIC EQUATIONS:}

Observations support homogeneous and isotropic model of the late universe, given by the lineelement [4]

$$
d s^{2}=d t^{2}-a^{2}(t)\left[d x^{2}+d y^{2}+d z^{2}\right]
$$


where $\mathrm{a}(\mathrm{t})$ is the scale factor.

In this space-time the standard Friedmann equation in GR is given by

$$
H^{2}=\frac{\kappa^{2}}{3} \rho
$$

where $\kappa^{2}=M_{P}^{-2}=8 \pi G$

On the brane in both RS-II and DGP model the conservation equation holds:

$$
\dot{\rho}+3 H(\rho+p)=0
$$

\section{A. Randall-Sundrum II Brane World}

In braneworld scenario, the gravity model is modified. In the space-time given by eq(2.1), RS-II model based Friedmann equation is obtained as [35, 52]

$$
H^{2}=\frac{\kappa^{2}}{3} \rho\left[1-\frac{\rho}{2 \lambda}\right]
$$

where $\lambda$ is the negative brane tension.

\section{B. Dvali-Gabadaze-Porrati Brane World}

Unlike the RS-II model, the infinite extra dimension is flat $\left(\Lambda_{5}=0\right)$ in DGP model. Brane tension is assumed to be zero or cancelled out with a brane cosmological constant. In the space-time given by eq(2.1), the DGP Friedmann equation is given by [40, 41]

$$
H^{2}=\left(\sqrt{\frac{\kappa^{2} \rho}{3}+\frac{1}{4 r_{c}^{2}}}+\epsilon \frac{1}{2 r_{c}}\right)^{2}
$$

or equivalently

$$
H^{2}-\epsilon \frac{H}{r_{c}}=\frac{\kappa^{2} \rho}{3}
$$

where $H=\frac{\dot{a}}{a}$ is the Hubble parameter, $\rho$ is the cosmic fluid energy density and $r_{c}=\frac{M_{P}^{2}}{2 M_{5}^{2}}$ is the crossover scale which determines the transition from $4 \mathrm{D}$ to $5 \mathrm{D}$ behavior and $\epsilon= \pm 1$.

For $\epsilon=1$, we have standard $\operatorname{DGP}(+)$ model which is self accelerating model without any form of dark energy, and effective $\omega$ is always non phantom. However for $\epsilon=-1$, we have DGP(-) model which does not self accelerate but requires dark energy on the brane. It experiences 5D gravitational modifications to its dynamics which effectively screen dark energy. 
In this paper we take DGP(-) model. Thus Friedmann eqs.(2.5) and (2.6) simplifies to

$$
H^{2}=\left(\sqrt{\frac{\kappa^{2} \rho}{3}+\frac{1}{4 r_{c}^{2}}}-\frac{1}{2 r_{c}}\right)^{2}
$$

and

$$
H^{2}+\frac{H}{r_{c}}=\frac{\kappa^{2} \rho}{3}
$$

\section{EFFECTIVE EQUATION OF STATE AND COSMIC EXPANSION :}

Here the nonlinear equation of state(EOS) for phantom fluid is taken as

$$
p=-\rho-f(\rho)
$$

where $f(\rho)=A \rho^{\alpha}$ i.e.,

$$
p=-\rho-A \rho^{\alpha}
$$

Connecting eqs.(2.3) and (3.2) we have

$$
\rho=\rho_{0}\left[1+3 \tilde{A}(1-\alpha) \ln \frac{a}{a_{0}}\right]^{\frac{1}{1-\alpha}}
$$

where $\alpha \neq 1$ and $\tilde{A}=A \rho_{0}^{\alpha-1}$. This EOS is suitable for cosmological data and centered around cosmological constant $\operatorname{EOS}(A=0)$. Parameters $A$ and $\alpha$ measures deviation from cosmological constant EOS. The sign of the parameter A determines whether DE is phantom or quintessence regime.

\section{A. Randall-Sundrum II Brane World}

Recently [6] we have analysed the EOS given by eq.(3.2) in RS-II model. A part of the following discussion is based on [6].

Connecting Friedmann eq(2.2) and conservation equation (2.3) [51, 52] we get

$$
\dot{H}+H^{2}=\frac{\ddot{a}}{a}=-\frac{\kappa^{2}}{2}(\rho+p)\left[1-\frac{\rho}{\lambda}\right]+\frac{\kappa^{2}}{6} \rho\left[1-\frac{\rho}{2 \lambda}\right]
$$

In GR-based theory, Friedmann equation is obtained as

$$
\dot{H}+H^{2}=\frac{\ddot{a}}{a}=-\frac{\kappa^{2}}{3}[\rho+3 P] .
$$


Comparing (3.4) and (3.5), the effective EOS with brane gravity corrections is obtained as

$$
P=-\rho-f(\rho)\left[1-\frac{\rho}{\lambda}\right]+\frac{\rho^{2}}{3 \lambda}
$$

using eq.(3.1).

Equation (3.6) yields

$$
\rho+P=-f(\rho)\left[1-\frac{\rho}{\lambda}\right]+\frac{\rho^{2}}{3 \lambda}
$$

and

$$
\rho+3 P=-2 \rho-3 f(\rho)\left[1-\frac{\rho}{\lambda}\right]+\frac{\rho^{2}}{\lambda}
$$

Here $f(\rho)=A \rho^{\alpha}$, therefore (3.6) yields

$$
P=-\rho-A \rho^{\alpha}\left[1-\frac{\rho}{\lambda}\right]+\frac{\rho^{2}}{3 \lambda}
$$

Equation (3.9) yields effective pressure $P<0$ for

$$
\rho<3 \lambda\left[1+A \rho^{(\alpha-1)}\left\{1-\frac{\rho}{\lambda}\right\}\right] .
$$

In particular, if we choose $\alpha=2$, then from eq(3.10) energy density must restricted as

$$
\frac{3 \lambda A-1-\sqrt{D_{\lambda}}}{6 A}<\rho<\frac{3 \lambda A-1+\sqrt{D_{\lambda}}}{6 A}
$$

where $D_{\lambda}=9 \lambda^{2} A^{2}-42 A \lambda+1$ and assuming $D_{\lambda}>0$

Further, it is found that

$$
\rho+P=-A \rho^{\alpha}\left[1-\frac{\rho}{\lambda}\right]+\frac{\rho^{2}}{3 \lambda}<0
$$

till

$$
\rho_{0}<\rho<3 \lambda A \rho^{(\alpha-1)}\left[1-\frac{\rho}{\lambda}\right]
$$

with $\rho_{0}$ being the present energy density.

This result shows that WEC will be violated till $\rho$ will satisfy the inequality (3.12). It will not be violated when

$$
\rho>3 \lambda A \rho^{(\alpha-1)}\left[1-\frac{\rho}{\lambda}\right] .
$$


This means that phantom fluid will behave effectively as phantom dark energy till $\rho$ will obey the inequality (3.12). It will not behave effectively as phantom when $\rho$ increases more and obeys the inequality (3.13).

Moreover, (3.8) shows that SEC will be violated till

$$
\rho<\lambda\left[2+3 A \rho^{(\alpha-1)}\left\{1-\frac{\rho}{\lambda}\right\}\right]
$$

This shows that when $\rho$ will increase such that

$$
3 \lambda A \rho^{(\alpha-1)}\left[1-\frac{\rho}{\lambda}\right]<\rho<\lambda\left[2+3 A \rho^{(\alpha-1)}\left\{1-\frac{\rho}{\lambda}\right\}\right]
$$

only SEC will be violated. It shows that when $\rho$ will satisfy the inequality (3.15), phantom characteristic to violate WEC will be suppressed by brane-gravity effects for negative brane tension and phantom fluid will behave effectively as quintessence. These results yield effective phantom divide at

$$
\rho=\rho_{\text {phd }}=3 \lambda A \rho_{\text {phd }}^{(\alpha-1)}\left[1-\frac{\rho_{\text {phd }}}{\lambda}\right] .
$$

It is interesting to see that even SEC will not be violated when

$$
\lambda\left[2+3 A \rho^{(\alpha-1)}\left\{1-\frac{\rho}{\lambda}\right\}\right]<\rho<2 \lambda .
$$

implying that, during the range (3.17), dark energy characteristic to violate SEC and WEC will be suppressed completely by brane-corrections in RS-II model.

Eqs.(2.3),(2.4) and (3.2) yield

$$
\dot{\rho}-3 A \sqrt{\frac{\kappa^{2}}{3}} \rho^{\alpha+\frac{1}{2}} \sqrt{1-\frac{\rho}{2 \lambda}}=0,
$$

where $\rho_{0} \leqslant \rho \leqslant 2 \lambda$.

Exact solution of this equation is obtained as

$$
\begin{gathered}
t=\frac{1}{\sqrt{3} \kappa A}\left[t_{0}+2(2 \lambda)^{(1 / 2)-\alpha}\left\{\sqrt{1-\frac{\rho_{0}}{2 \lambda}} 2 F_{1}\left(\frac{1}{2}, \frac{1}{2}+\alpha, \frac{3}{2}, 1-\frac{\rho_{0}}{2 \lambda}\right)\right.\right. \\
\left.\left.-\sqrt{1-\frac{\rho}{2 \lambda}} 2 F_{1}\left(\frac{1}{2}, \frac{1}{2}+\alpha, \frac{3}{2}, 1-\frac{\rho}{2 \lambda}\right)\right\}\right],
\end{gathered}
$$

where $2 F_{1}(a, b, c, x)$ is the hypergeometric function.Further, using (3.19) in (3.3), we get a relation between time $t$ and the scale factor $a(t)$.

As maximum value of $\rho$ is $2 \lambda$, so phantom universe will expand upto time $t_{m}$ given as

$$
t_{m}=\frac{1}{\sqrt{3} \kappa A}\left[t_{0}+2(2 \lambda)^{(1 / 2)-\alpha} \sqrt{1-\frac{\rho_{0}}{2 \lambda}} 2 F_{1}\left(\frac{1}{2}, \frac{1}{2}+\alpha, \frac{3}{2}, 1-\frac{\rho_{0}}{2 \lambda}\right)\right]
$$


with $t_{0}$ being the present time. Moreover, from (3.3), it is obtained that

$$
3(1-\alpha) A \ln \left(a_{m} / a_{0}\right)=(2 \lambda)^{1-\alpha}-\rho_{0}^{1-\alpha},
$$

where $a_{m}=a\left(t_{m}\right)$. This equation shows that if $\alpha \gtrless 1,2 \lambda>\rho_{0}$ as $a_{m}>a_{0}$.

From (3.16) and (3.19), we obtain effective phantom divide at time

$$
\begin{gathered}
t=t_{\text {phd }}=\frac{1}{\sqrt{3} \kappa A}\left[t_{0}+2(2 \lambda)^{(1 / 2)-\alpha}\left\{\sqrt{1-\frac{\rho_{0}}{2 \lambda}} 2 F_{1}\left(\frac{1}{2}, \frac{1}{2}+\alpha, \frac{3}{2}, 1-\frac{\rho_{0}}{2 \lambda}\right)\right.\right. \\
\left.\left.-\sqrt{1-\frac{\rho_{\text {phd }}}{2 \lambda}} 2 F_{1}\left(\frac{1}{2}, \frac{1}{2}+\alpha, \frac{3}{2}, 1-\frac{\rho_{\text {phd }}}{2 \lambda}\right)\right\}\right],
\end{gathered}
$$

Inequalities (3.15) and (3.17) show that for $\rho$ satisfying

$$
\rho<\lambda\left[2+3 A \rho^{(\alpha-1)}\left\{1-\frac{\rho}{\lambda}\right\}\right]
$$

SEC will be violated. It means that the universe will accelerate till $\rho$ will obey (3.23). But as $\rho$ will grow more and it will satisfy

$$
\rho>\lambda\left[2+3 A \rho^{(\alpha-1)}\left\{1-\frac{\rho}{\lambda}\right\}\right]
$$

SEC will not be violated. It means that the universe will decelerate when $\rho$ will obey the inequality (3.24). It shows a transition from acceleration to deceleration at $\rho=\rho_{\text {tr }}$ given by the equation

$$
\rho_{\mathrm{tr}}=\lambda\left[2+3 A \rho_{\mathrm{tr}}^{(\alpha-1)}\left\{1-\frac{\rho_{\mathrm{tr}}}{\lambda}\right\}\right]
$$

Connecting (3.19) and (3.25), we obtain that this transition will take place at time

$$
\begin{gathered}
t=t_{\mathrm{tr}}=\frac{1}{\sqrt{3} \kappa A}\left[t_{0}+2(2 \lambda)^{(1 / 2)-\alpha}\left\{\sqrt{1-\frac{\rho_{0}}{2 \lambda}} 2 F_{1}\left(\frac{1}{2}, \frac{1}{2}+\alpha, \frac{3}{2}, 1-\frac{\rho_{0}}{2 \lambda}\right)\right.\right. \\
\left.\left.-\sqrt{1-\frac{\rho_{\mathrm{tr}}}{2 \lambda}} 2 F_{1}\left(\frac{1}{2}, \frac{1}{2}+\alpha, \frac{3}{2}, 1-\frac{\rho_{\mathrm{tr}}}{2 \lambda}\right)\right\}\right] .
\end{gathered}
$$

\section{B. Dvali-Gabadaze-Porrati Brane World}

At high energy (early universe) $\frac{1}{r_{c}}$ is small and can be neglected, therefore from eq(2.7), we obtain the standard GR Friedmann equation as

$$
H^{2}=\frac{\kappa^{2}}{3} \rho
$$


In the late universe, the extra dimension effect cannot be neglected and we shall use a approximation form of Friedmann equation [56] as follows.

The DGP Friedmann equation (2.7) can be written as

$$
H^{2}=\frac{1}{4 r_{c}}\left[\sqrt{1+\frac{4 \rho r_{c}^{2}}{3 M_{P}^{2}}}-1\right]^{2}
$$

Expanding in terms of $\rho r_{c}^{2} / M_{P}^{2} \ll 1$, we get at the lowest order

$$
H \approx \frac{\kappa^{2} r_{c}}{3} \rho
$$

In this case, using (2.3) and (3.2), we have

$$
\dot{H}=\frac{\kappa^{4} r_{c}^{2} A}{3} \rho^{\alpha+1}
$$

Consequently

$$
\dot{H}+H^{2}=\frac{\ddot{a}}{a}=\frac{\kappa^{4} r_{c}^{2}}{3}\left(A \rho^{\alpha+1}+\frac{\rho^{2}}{3}\right)
$$

Comparing this equation with GR based Friedmann equation (3.5), the effective EOS in this case is obtained as

$$
P=-\rho-\frac{2}{3} \kappa^{2} r_{c}^{2} \rho^{2}\left(\rho^{\alpha-1}+\frac{1}{3}\right)+\frac{2}{3} \rho
$$

Equation (3.32) yields that

$$
\rho+P=\frac{2}{3} \rho-\frac{2}{3} \kappa^{2} r_{c}^{2}\left(A \rho^{\alpha+1}+\frac{\rho^{2}}{3}\right)
$$

and

$$
\rho+3 P=-2 \kappa^{2} r_{c}^{2} \rho^{2}\left(A \rho^{\alpha-1}+\frac{1}{3}\right)
$$

From eq(3.34) we see that,unlike RS-II model , SEC is always violated in this case.Further from $(3.33)$

$$
\rho+P=\frac{2}{3} \rho-\frac{2}{3} \kappa^{2} r_{c}^{2}\left(A \rho^{\alpha+1}+\frac{\rho^{2}}{3}\right)<0
$$

till

$$
\rho_{0}<\rho<\kappa^{2} r_{c}^{2}\left(A \rho^{\alpha+1}+\frac{\rho^{2}}{3}\right)
$$


with $\rho_{0}$ being the present energy density. In particular, if we choose $\alpha=2$, then for violation of WEC, the energy density must be restricted as

$$
\rho>\frac{-1+3 \sqrt{D_{r_{c}}}}{6 A}
$$

where $D_{r_{c}}=\frac{1}{9}+\frac{4 A}{\kappa^{2} r_{c}^{2}}$

The parameter $\tilde{A}$ in eq (3.3) determines the type of behaviour of the DE density with the expansion of the universe. The speed of change of the DE density is given by

$$
\frac{d \rho}{d a}=3 \tilde{A} \rho_{0}\left[1+3 \tilde{A}(1-\alpha) \ln \frac{a}{a_{0}}\right]^{\frac{1}{1-\alpha}-1}
$$

This shows that for $\tilde{A}>0$, DE density grows, for $\tilde{A}=0$, the dark energy is constant while for $\tilde{A}<0$, it decreases with the expansion of the universe. Thus for $\tilde{A}>0$, phantom energy increases with expansion and there will be a time when

$$
\rho>\kappa^{2} r_{c}^{2}\left(A \rho^{\alpha+1}+\frac{\rho^{2}}{3}\right)
$$

For $\alpha=2$, in this case energy density is restricted as

$$
\rho<\frac{-1+3 \sqrt{D_{r_{c}}}}{6 A}
$$

This shows that WEC will be violated till $\rho$ satisfies the inequality (3.35). It will not be violated when $\rho$ satisfies (3.37). It means that when $\rho$ satisfies the equality (3.37) the phantom character of violating WEC will be suppressed by brane-gravity effects and phantom fluid will behave effectively as quintessence. This yields the effective phantom divide at

$$
\rho=\rho_{\mathrm{phd}}=\kappa^{2} r_{c}^{2}\left(\tilde{A} \rho_{0}^{1-\alpha} \rho_{\mathrm{phd}}^{\alpha+1}+\frac{\rho_{\mathrm{phd}}^{2}}{3}\right)
$$

Connecting MFE(modified Friedmann equation) (3.29) and conservation equation (2.3) we have

$$
t=t_{0}-\frac{1}{\kappa^{2} \alpha \tilde{A} r_{c} \rho_{0}^{1-\alpha}}\left(\rho^{-\alpha}-\rho_{0}^{-\alpha}\right)
$$

Using eqs(3.38) and (3.39), we get the effective phantom divide at time

$$
t_{\mathrm{phd}}=t_{0}-\frac{1}{\kappa^{2} \alpha \tilde{A} r_{c} \rho_{0}^{1-\alpha}}\left(\rho_{\mathrm{phd}}^{-\alpha}-\rho_{0}^{-\alpha}\right)
$$

It is to be noted that in GR in order to have phantom crossing EOS needs to have double value [55] i.e., $p=-\rho \pm f(\rho)$ 
The eq. (3.39) can be written as

$$
\rho=\rho_{0}\left[1-\kappa^{2} \alpha \tilde{A} r_{c} \rho_{0}\left(t-t_{0}\right)\right]^{-\frac{1}{\alpha}}
$$

which can be rewritten as

$$
\rho=\frac{3 H_{0}^{2} \Omega_{0}}{\kappa^{2}}\left[1-\frac{3 \tilde{A} \alpha \Omega_{0} H_{0}}{2 c}\left(t-t_{0}\right)\right]^{-\frac{1}{\alpha}}
$$

where

$$
\Omega_{0}=\frac{\kappa^{2} \rho_{0}}{3 H_{0}^{2}} \quad \text { and } \quad \Omega_{r_{c}}=\frac{1}{4 r_{c}^{2} H_{0}^{2}}
$$

are the usual dimensionless density parameters.

In terms of these parameters Friedmann eq (2.8) can be written as

$$
\Omega_{0}+2 \sqrt{\Omega_{r_{c}}}=1
$$

Solving (3.29) and (3.42) the scale factor in this case is obtained as

$$
a(t)=a_{0} \exp \left[\frac{1}{6 \tilde{A}(\alpha-1) \sqrt{\Omega_{r_{c}}}}\left(1-\frac{3 \tilde{A} \alpha \Omega_{0} H_{0}\left(t-t_{0}\right)}{2 \sqrt{\Omega_{r_{c}}}}\right)^{\frac{-1}{\alpha}}\left(3 \tilde{A} \alpha \Omega_{0} H_{0}\left(t-t_{0}\right)-2 \sqrt{\Omega_{r_{c}}}\right)+2 \sqrt{\Omega_{r_{c}}}\right]
$$

This is the expression of the scale factor at late stages of evolution when $\rho$ is very small. Note that for $\alpha>1, a(t)$ grows exponentially with time while for $\alpha \leqslant 1, a(t)$ becomes constant asymptotically i.e., we shall have a static model of the universe. Thus for $\alpha>1$, the late stage acceleration as demanded by the present day observation is obtained while for $\alpha \leqslant 1$, the universe expands to a (finite) maximum volume and then becomes static asymtotically.

\section{CONCLUSIONS:}

In this paper we analyze and compare behaviour of phantom fluid in DGP model (normal branch) of brane gravity with RS-II model having negative brane tension lambda. Brane corrections make drastic changes to the behaviour of phantom fluid which is characterised by violation of WEC and accelerating the universe ending up in big-rip singularity in most of the models. 
In both DGP and RS-II model the phantom characteristic of violating WEC is suppressed by brane gravity effects. Moreover both models are free from big-rip problem. In RS-II model brane tension plays a crucial role. When it is negative, it causes drastic changes in the behaviour of dark energy, but phantom fluid has usual behaviour when brane tension is positive.

In DGP model SEC is always violated, consequently there is acceleration only. Contrary to this in RS-II model acceleration is transient showing the non violation of even SEC. In RS-II model the future universe begins at time $t_{0}$, the present age of the universe, and it stops expanding when energy density grows to $2 \lambda$ by time $t_{m}$. Contrary to this in DGP model there is no maximum limit of expansion.

\section{Acknowledgement:}

A part of the paper is done during a visit to IUCAA, Pune, India. The first author is thankful to IUCAA for warm hospitality and facility of doing research works.

[1] Perlmutter, S. J. et al.(1999), Astrophys. J. 517,565; (1998) astro-ph/9812133.

[2] Spergel, D. N. et al,(2003), Astrophys J. Suppl. 148 175[ astro-ph/0302209] and references therein.

[3] Riess, A. G. et al,(2004), Astrophys. J. 607, 665 [astro-ph/0402512.

[4] Miller, A.D. et al ,(1999), Astrophys. J. Lett. 524 L1.

[5] E.J.Copeland, M.Sami and S. Tsujikawa, Int. J. Mod. Phys. D, 15,(2006)1753 hep-th/0603057] and references therein.

[6] Srivastava, S.K. and Dutta, J.,(2009) Int. J. Mod. Phys. D ,18(2), 329 [arXiv:0708.4189 (gr-qc)].

[7] Caldwell, R.R.(2002), Phys.Lett.B,545,23.

[8] Caldwell, R.R., Kamionkowski, M. and Weinberg, N.N.(2003), Phys. Rev. Lett., 91, 071301.

[9] Overduin, J.M., Cooperstock, F.I., (1998), Phys. Rev. D 58, 043506.

[10] Armendariz-Picon, C., Damour,T. and Mukhanov,V. ,(1999), Phys. Lett. B 458, 209.

[11] Chiba,T., Okabe, T. and Yamaguchi, M. ,(2000), Phys. Rev. D 62, 023511.

[12] Sen, A. , (2002), J. High Energy Phys. 04, 048. (2002) 07, 065; (2002), Mod. Phys. Lett. A 17, (2002)1799.

[13] Sen, A. ,(2002) 07, 065; (2002), Mod. Phys. Lett. A 17, (2002)1799.

[14] Garousi, M. R., (2000),Nucl. Phys. B 584, 284;(2003) J. High Energy Phys. 04, 027. 
[15] Singh, Parampreet ,Sami, M., Dadhich, Naresh (2003) ,Phys.Rev. D 68, 023522 and references therein.

[16] Srivastava, S.K.,(2004), gr-qc/040974.

[17] Sami, M. , Toporensky, A., Tretjakov, P.V., Tsujikawa, S.(2005), Phys. Lett. B 619193 hep-th/0504155.

[18] Calcagni, G., Tsujikawa, S., Sami, M.,(2005), Class. Quan. Grav. 223977 [hep-th/0505193].

[19] Srivastava, S.K.; (2005), Phys. Lett. B 6191 astro-ph/0407048.

[20] Jackiw, R. (2000), physics/0010042.

[21] Bertolami, O. et al, (2004),Mon. Not.R.Astron.Soc. 353329 astro-ph/0402387.

[22] Bento, M. C. , Bertolami,O. ,Sen, A.A., (2002),Phys. Rev. D 66043507 gr-qc/0202064.

[23] Capozziello, S.,(2002), Int.J.Mod.Phys.D 11483.

[24] Capozziello, S., Carloni,S., Troisi, A., (2003), astro-ph/0303041.

[25] Caroll, S.M., Duvvuri,V., Trodden, M., Turner, M.S.,(2004), Phys.Rev. D 70043528, astro-ph/0306438.

[26] Dolgov,A.D., Kawasaki,M.,(2003), Phys.Lett.B 5731 ; astro-ph/0307285.

[27] Soussa, M.E., Woodard, R.P.,(2004), Gen. Relativ. Gravit. 36 855, astro-ph/0308114.

[28] Nojiri, S., Odintsov, S.D.,(2004), Phys.Lett.A 19(2004)627; hep-th/0310045.

[29] Nojiri, S., Odintsov, S.D., (2003), Phys.Rev. D 68123512, hep-th/0307228

[30] Abdalaa, M.C.B., Nojiri, S., Odintsov, S.D.,(2005), Class. Quan. Grav. 22 L35, hep-th/0409117

[31] Mena,O., Santiago, J., Weller, J.,(2006), Phys.Rev.Lett. 96(2006)041103.

[32] Atazadeh, K. ,Sepangi, H.R.(2006),, gr-qc/0602028.

[33] Bouhmadi-Lopez, M. et al,(2005), astro-ph/0512124; (2006) gr-qc/0612135; (2007) arXiv:0707.2390; (2007) arXiv:0706.3896 astro-ph].

[34] Rubakov, V. A.,(2001), Phys. Usp. 44, 871 hep-ph/0104152].

[35] Maartens, R.,(2004) Lving Rev. Relativity, 7, 7, [(2003) gr-qc/0312059].

[36] Brax, P. et al,(2004), Rep. Prog. Phys. 67, 2183 hep-th/0404011.

[37] Csa'ki, C.,(2004) hep-ph/0404096

[38] Randall,L., Sundrum, R.,(1999),Phys. Rev. Lett., 83, 3370 .

[39] Randall,L., Sundrum, R.,(1999),Phys. Rev. Lett., 83, 4690.

[40] Dvali,G.R. , Gabadadze,G. and Porrati, M. (2000), Phys.Lett. B 485208 [hep-th/000506].

[41] Deffayet,D. (2001), Phys.Lett. B 502199.

[42] Deffayet,D., Dvali,G.R. and Gabadadze,G. (2002), Phys.Rev.D 65044023 astro-ph/0105068.

[43] Bine'truy, P., Deffayet, C., Langlois, D.,(2000), Nucl. Phys. B 565, 269 hep-th/9905012

[44] Bine'truy, P., Deffayet, Ellwanger, U., C., Langlois, D.,(2000) Phys. Lett.B 477,289 hep-th/9910219].

[45] Csa'ki, C., Graesser, M., Kolda, C., Terning,(1999) J., Phys. Lett.B 462, 34 hep-ph/9906513. 
[46] Cline, J.M., Grojean, C., Servant, G.,(1999), Phys. Rev. Lett. 83 , 4245 hep-ph/9906523.

[47] Maartens, R., Wands, D., Bassett, B.A., Heard, I.P.C., (2000),Phys. Rev. D 62, 023509 hep-ph/9912464.

[48] Copeland, E.J., Liddle, A.R., Lidsey, J.E., (2001), Phys. Rev. D 64, 041301 astro-ph/0006421]

[49] Calcagni, G.,(2004), Phys. Rev. D 69, 103508.

[50] Apostolopoulous, P.S., Tetradis, N., (2006)Phys. Lett.B 633, 409.

[51] Nojiri, S., Odintsov, S.D.,(2004), Phys. Lett. B, 595, 1 [(2004) hep-th/0408170.

[52] Srivastava, S.K., (2007), Gen. Rel. Grav., 39, 241.

[53] Nojiri, S., Odintsov, S.D., Tsuijikawa, S.,(2005), Phys. Rev. D, 71, 063004.

[54] Štefančic, H.,(2005) Phys. Rev. D, 71, 084024.

[55] Nojiri, S., Odintsov, S.D.,(2005), Phys. Rev. D, 72, 023002.

[56] Gumjudupai, Burin.,(2004), Gen. Rel. Grav., 36, 747. 